\title{
Why use predictive processing to explain psychopathology? The case of anorexia nervosa
}

\author{
Stephen Gadsby and Jakob Hohwy \\ Cognition \& Philosophy Lab \\ Department of Philosophy \\ Monash University
}

To appear in: The Philosophy and Science of Predictive Processing (Eds.) S. Gouveia, R. Mendonça, \& M. Curado. Bloomsbury.

\begin{abstract}
Predictive processing accounts are increasingly called upon to explain mental disorder. They seem to provide an attractive explanatory framework because the core idea of prediction error minimization can be applied to simultaneously account for several perceptual, attentional and reasoning deficits often implicated in mental disorder. However, it can be unclear how much is gained by such accounts: the proffered explanations can appear to have several weaknesses such as being too liberal, too shallow, or too wedded to formal notions of statistical learning. Here, we taxonomise the relatively unrecognised variety of explanatory tools under the framework and discuss how they can be employed to provide substantial explanations. We then apply the framework to anorexia nervosa, an eating disorder that is characterised by a complex set of perceptual, reasoning and decision-making problems. We conclude that the predictive processing framework is a valuable type of explanation for psychopathology.
\end{abstract}

Keywords: predictive processing, mental disorder, anorexia nervosa, perceptual inference, active inference, precision optimization, interoception, body size experience

\section{Introduction}

Predictive processing is a framework for explaining mental function, which is gaining increasing influence in cognitive science (Hohwy 2013, Clark 2016). PP accounts are increasingly called upon to explain mental disorder. It seems an attractive explanatory framework because the core idea of prediction error minimization can be applied to simultaneously account for several perceptual, attentional and reasoning deficits.

However, it can seem unclear how much is truly gained by such accounts: the proffered explanations appear to have several weaknesses such as being (i) too liberal, since PP is a framework for approximating Bayesian inference and any behaviour can be fitted to some prior and likelihood function or cost function, (ii) too shallow, since PP accounts often merely subsume or re-describe existing theories or explanations, or (iii) too wedded to formal notions of statistical learning, in the sense that psychopathology reduces to failures of absorbing statistical information, rather than to biologically based illness.

Here, we first focus on the often unrecognised variety of explanatory tools under the PP framework and discuss how they can be employed to provide substantial explanations. We then apply the framework to anorexia nervosa (AN), an eating disorder characterised by a complex set of perceptual, reasoning and decision-making problems. We conclude that the 
PP framework is a valuable type of explanation for psychopathology. It provides advances in understanding because it can make new distinctions within existing approaches to AN and provide new understanding of some key problems for these approaches, such as the resistance of patients' pathological belief to counterevidence. The key contribution is that, under PP, the only imperative for an agent is to minimize prediction error in the long-term average, by any tools available; often those tools are quite abstract priors about model selection, simplicity and types of policies for action over different time scales, about which it is difficult to be confident. This predicts individual differences in different contexts, some of which can be associated with mental illness.

\section{Predictive Processing and Psychopathology}

Predictive processing approaches to mind and cognition belong to a broad tradition of views centred on internal generative models of the causes of sensory input, or, more heuristically, perceptual inference. In its contemporary shape, PP posits that internal models are used to generate predictions of the sensory input that would occur, if the environment (including the agent) is as the model describes. Predictions are then tested against the actual sensory input such that evidence for the model is maximised for accurate and precise predictions. The initial upshot of such PP is veridical perception of the causes of sensory input. The basic framework is reviewed extensively elsewhere (see Hohwy 2013, Clark 2016; for introduction to the mathematical and computational background see Bogacz 2017, Buckley, Kim et al. 2017). Here, we discuss several more intricate properties of PP, in particular associated with the active inference approaches to PP, associated with the free energy principle and process theories under this principle (Friston 2010). This discussion then sets the scene for discussion of PP approaches to psychopathology, in particular our test case: anorexia nervosa.

Perceptual inference is inference of the causes of sensory input. Under PP (in particular when implemented with predictive coding as the process theory), this is not merely a matter of matching predictions directly with input. The difference between predictions and input is the prediction error, which functions as a learning signal in the revision of the internal model. The amount of learning accrued from prediction error can be conceived as the learning rate (cf. Bayesian inference). The learning rate is governed by the ratio of the likelihood precision (i.e., the precision of the prediction error) and the prior precision (i.e., the degree of existing evidence for the model generating the prediction). The learning rate is variable, under contextually guided expectations for the volatility of the sensory input (i.e., unexpected changes in the mean and precision of the input). This ensures that the perceptual system in question can engage adaptively within a dynamically changing environment, characterised by uncertainty and non-linearities in the sensory input. The range of inferred causes is broad, encompassing both the external environment and the internal environment of bodily states (i.e., proprioception, interoception).

Perceptual inference through predictive coding is hierarchical, which reflects both the interaction of contextual precision beliefs with the precision gain set on sensory input, and the deep causal structure of the sensorium; thus, many different levels of the perceptual hierarchy contribute to everyday perception of bodies, houses, cars, trees and the other objects and states in and around us. One organising principle for the hierarchy is spatiotemporal scale, where higher levels have wider receptive fields and process causal regularities occurring over longer time scales. In this perspective, different psychopathologies can be phenotyped in terms of different constellations of priors (Montague, Dolan et al. 2012, Friston, Redish et al. 2017). For example, autism has been hypothesised to relate to a strong 
prior expectation for the precision of sensory input (Van de Cruys, Evers et al. 2014) or a strong prior belief in low volatility of sensory input (Palmer, Lawson et al. 2017); psychotic symptoms associated with schizophrenia, such as hallucinations, have been linked with different volatility beliefs as well (Sterzer, Adams et al. 2018, Corlett, Horga et al. 2019).

Just as perception is inferential, so is action. Most fundamentally, adaptive agents need to select optimal policies (conceived as sequences of control states leading to concrete behaviours) to achieve their desired (expected) states, a task that is made difficult in a dynamic environment where there can be no one-one relation between policies and outcomes. This therefore presents a forward-looking inference problem: which policy should the agent infer is its own? Inference of policies then leads to action as the inferred policy induces prediction error that is minimized by selectively sampling sensory input expected under that policy. This is known as active inference.

As with perceptual inference, precisions are crucial for active inference; under an expectation that the agent occupies states with efficient prediction error minimization, high precision policies are favoured (i.e., policies that have high fidelity mapping from actions to observed outcomes). Policies are thus ranked on their precision, which captures how confident the agent can be that they will produce states that are not far removed from the expected states of the agent (i.e., the (Kullbach-Leibler) divergence between the state that the policy can be expected to achieve and the expected state). Active inference comes in two flavours: there is action for utility, which is the common notion that we act to reap rewards, and then there is action for epistemic value, which is the less common idea that we act to reduce uncertainty about our model of the world (e.g., looking closer to see if that dark, moving object is really a spider). Thus, sometimes one must act to reduce uncertainty about the world in order to get oneself into a position where one can act to reap rewards (Friston, Rigoli et al. 2015, Friston, FitzGerald et al. 2016).

Across perceptual and active inference, $\mathrm{PP}$ is subject to one critical imperative, namely that prediction error should be minimized over the long-term average. That is, PP is not a simple rule that only the current prediction error is minimized. If that were the case, then shutting down all systems and sitting in a dark room would be the most efficient strategy. But, of course, we don't do that, precisely because that strategy would lead to increased deviation from our expected states in the longer term, as we get hungry and dirty, as we don't get paid because we don't turn up to work, and so on. Rather, we operate within expected boundaries of prediction error, allowing us to maintain ourselves within our expected states in the longterm. This is reflected in our inference of policies, as some actions will be, for example, aimed at exploring the current state, even if that might temporarily increase prediction error.

The models on which perceptual and active inference are based should be neither too simple nor too complex. Overly simple models cannot accommodate future contingencies, produced by interactions among hidden causes, and overly complex models are fitted to noise that does not repeat - thus in both cases the models will generate more prediction error than they would if their complexity had been optimised. Model complexity is non-trivial to get right, and depends on longer-term learning of levels of irreducible noise and of patterns of statedependent noise in different contexts. It is common to consider issues of complexity for perceptual inference, but it is critical for active inference too. The expected prediction error under a policy depends on the complexity of the underlying model, such that execution of an overly cumbersome policy in a messy environment may be less productive than a simpler policy, even if the former would give an excellent outcome were it to succeed. This in turn 
speaks to beliefs about the controllability of the (volatile) environment as well as issues around the time scale over which policies are relevant.

Simplicity and complexity relate to (Bayesian) model selection in a wider sense, where simplicity may be a factor alongside context-dependence considerations. Model selection can be considered a discrete process, where a given model is prioritised (e.g., this is a dice game rather than a coin throwing game) and prediction error then minimized under that model. Selection of a model is revisited if there is unexpected accumulation of prediction error under the model, forcing selection of a new model. This process is however sensitive to the prior probability of competing models; in principle, if no alternative model has significant model evidence (e.g., competing models are overly complex), then the system in question may need to tolerate quite high levels of accumulated prediction error and thus sub-optimal inference.

Beliefs, thoughts, and conscious perceptions are the upshots of the underlying perceptual and active predictive processes. Bayesian priors are sometimes labelled 'belief' but should not be confused with explicit, propositional belief. Exactly why belief, thought and perceptions have the phenomenology that they do is not strongly determined by accounts of PP. However, it does seem likely that the structure of experience is somehow determined by the process of prediction error minimization. For example, in the well-known rubber hand illusion, touch sensation and sense of ownership is felt for a visible but fake hand when the real, hidden hand is touched synchronously with the fake hand. The mislocation of touch may be driven by weighting of expected precisions in the sensory input, and favouring of the illusory hypothesis may be facilitated by complexity considerations, as the veridical hypothesis, (namely that the experimenter is the hidden cause of both the seen touch and the unseen touch on the real hand) has a more complex causal structure (Hohwy 2013: Ch 5-6). It is not commonly observed that similar false inference can occur in the domain of active inference too. Thus we should expect that, in some circumstances, expectations of precision conspire with particular contexts and complexities to lead to false inference of policies.

Overall, the PP framework provides a picture of perceptual and cognitive processes that goes substantially beyond the initial idea of just a meeting of predictions and input. There are many interlocking predictive processes that prepare the ground for, and execute, successful long-term average prediction error minimization. Since no environment is ever learned completely, priors about precisions, complexities, volatilities and irreducible noise will be fraught with uncertainty. This predicts that there will be individual differences in how these processes are balanced against each other. More abstractly, under a variational inference scheme, there is approximation to exact inference as the different parameters of the recognition model are optimised; this means the inference will spend time deviating from the optimal, exact inference, which will manifest as more or less transitory or entrenched false inference.

The richness of the PP toolbox mitigates somewhat against several perceived weaknesses of its use to account for mental disorder. We briefly consider three of these perceived weaknesses. PP is often considered too liberal because any behaviour can be "explained" in Bayesian terms by picking appropriate priors and likelihoods (Jones and Love 2011). However, under the complete class theorem (which guarantees this situation), this is now conceived as a strength. This is implicit in the comment above, to the effect that psychopathologies can be phenotyped down to an individual level according to their priors, derived from the behaviour. This yields a principled, unifying nosology expressed in PP terms. Admittedly, this leads to a second perceived weakness, namely that now explanations 
are too shallow. The worry is that even though psychopathology can be phenotyped mathematically through their priors, there is no guarantee as to how these priors are expressed in terms of real, underlying neuronal mechanisms. Explaining psychopathology in terms of priors seems merely descriptive. However, this weakness can be overcome by identifying anatomically informed models, which locate the offending priors and the associated message passing along neuronal pathways, subject to specific neurotransmitter processes appropriate to the different computational roles needed for PP (Marshall, Mathys et al. 2016, Parr and Friston 2018).

Finally, by phrasing everything in terms of probabilistic inference, it may appear that PP is too wedded to statistical learning, in the sense that every intractable mental disorder appears to be a mere failure of absorbing the right statistical regularities, and subject to correction through education on statistical regularities. However, explaining psychopathologies in terms of errors in statistical learning highlights the possibility that departures from optimal perceptual and active inference can fail to correct themselves over time, as they lead to cascading and increasingly ensconced malfunctions; this may capture the crucial developmental aspect of many disorders. Beyond this, anatomically informed models show how intractable (chronic) malfunction is reflected in the computational roles necessary for the various aspects of optimal PP. Given these models, biological malfunction can be seen as the underlying cause of failures in statistical learning.

The PP framework thus appears potentially resourceful as a general approach to psychopathology, though there remain several unresolved questions. In the remainder of this chapter, we consider anorexia nervosa under the PP framework. This will yield both a new take on this disorder, and serve as a test case for the explanatory worth of PP in psychopathology.

\section{The empiricist model of anorexia nervosa}

While predictive coding's explanatory toolkit has been applied to a host of psychopathologies, here we consider its application to a further disorder: anorexia nervosa (AN). We focus first on a centrally relevant feature of the disorder: the false beliefs patients hold about their own body size. Despite their dangerously low weight, AN patients' regularly profess to being overweight, or at least not being excessively thin (Gadsby 2017a). A first step in understanding how the PP framework might illuminate the cause of such beliefs is in reviewing explanations that have already been provided.

Recently, an empiricist model of these beliefs has been offered (Gadsby 2017a; 2017b). The guiding insight of empiricist models - commonly associated with Maher's (1974) treatment of delusions - is that the proximal cause of delusional beliefs are particularly unusual experiences that patients suffer from. The empiricist model of false body size beliefs in anorexia thus specifies a number of abnormal experiences of body size that patients may suffer from. Here, we focus on two of the abnormal experiences of body size posited by this model (for more discussion, see Gadsby 2018).

One proposal rests on evidence suggesting that AN patients suffer from what is known as recurrent spontaneous mental imagery. Most commonly associated with social anxiety disorder, this phenomenon involves individuals experiencing conscious, invasive, recurrent mental imagery representing their fears (i.e. having an overweight body) (Kadriu et al. 2019). One eating disordered patient describes the content of one such imagery experience, "In the 
image I see myself obese, with very fat thighs and a fat stomach." (ibid., p. 8). In the case of social anxiety disorders, patients are known to interpret this imagery as veridical, coming to believe that the content of these images episodes accurately reflects reality. The empiricist model of anorexia suggests that if a similar interpretational bias is present in $\mathrm{AN}$, then these episodes of spontaneous imagery may be the cause of the relevant false body size beliefs (Gadsby 2018).

A second proposal for a form of abnormal experience of body size relates to the process of self-other comparison. Specifically, it has been suggested that due to a distorted mental representation of their own bodies (the "perceptual body image"), patients engage in dysfunctional self-other body comparison (Gadsby 2017a). When assessing whether other seen bodies are either larger or smaller than themselves, they perceptually misjudge, such that bodies which are in fact larger are judged to be smaller. These consistent misjudgementsi.e. "my body is bigger than hers" - are proposed to ground the relevant false body size beliefs.

How might the PP framework help to illuminate and contribute to this explanatory model? In keeping with the PP account of delusional beliefs as arising in response to aberrant prediction error (Fletcher and Frith 2009), we might posit that the relevant false beliefs about body size emerge through an attempt to "explain away" ongoing aberrant prediction error concerning body size. However, it is not immediately clear that the PP framework in itself brings any explanatory benefit, above and beyond the standard empiricist model — whether we use the term "abnormal experience" or "aberrant prediction error" apparently makes little difference to the mechanistic details of the model itself.

Building on the outline of PP in the previous section, we do believe there can be positive explanatory advantage to redescribing the empiricist approach in PP terms. The framework can point to a mapping of empiricist or folk psychological idioms onto the parts and processes of a neural message passing mechanism. For example, if one relies on expectations of precisions as well as of means, then PP can illuminate their interaction in Bayesian as well as mechanistic terms, as there must be distinct neuronal pathways for the message passing of both precisions and means. This is not anticipated on the standard empiricist approach, and could help explain, for example, more subtle aspects of AN experience relating to multisensory integration (Case, Wilson, \& Ramachandran, 2012; Zopf et al., 2016). In addition, once we consider the PP mechanism, new explanatory avenues become apparent, for example, relating to the level of the hierarchy at which deficits arise or cascade to, and to ways in which cognitive or pharmacological interventions may be expected to work (for example, a cognitive learning task that engages volatility learning may be more appropriate than a task that engages learning of means) (Parr, Benrimoh et al. 2018; Tulver et al. 2019).

The PP account can also generate new conceptual and mechanistic distinctions among the proposed types of experience of body size in the empiricist account. For example, imagery concerns the upshot of perceptual inference, under conditions where the bottom-up signal is weighted low; low weighting of bottom-up signals would relate to precision optimisation, which can either be exogenous (driven by the precision of the bottom-up prediction error signal) or endogenous (volitional reduction of expected precision of sensory input, cf. attention) (for a relevant PP account, see Aru et al 2018). Spontaneous imagery of body size would then arise through perceptual inference that is poorly controlled by the bottom-up prediction error signal, possibly partly driven exogenously by poor precision of the interoceptive prediction error signals in AN. 
While faulty self-other comparison is presumed to stem from the misrepresentation of bodily dimensions (Gadsby, 2017c), PP may illuminate how this misrepresentation arises. A key insight of the PP framework is that misrepresentation (i.e. inaccurate means) can arise due to supoptimal estimates of prediction error precision (Fletcher \& Frith, 2009). Perhaps the imprecision of bodily signals in AN (Herbet \& Pollatos, 2018) is responsible for the misrepresentation of bodily dimensions which, in turn, causes the evident dysfunctional selfother body comparisons.

It is worth noting that though these two potential analyses of the empiricist approach are distinct, they both refer to differences in precision optimization for body size in AN. This may serve to unify the otherwise distinct accounts in one proposal, namely that imprecision is involved in some of the unusual experiences of body size cited in the empiricist account. One possible hypothesis would then be that the primary processing problem is not in the unusual experiences themselves, and the misrepresentation of bodily dimensions they suggest, but in precision optimization. This hypothesis would be aligned with PP accounts of other disorders, which also revolve around precision optimisation (e.g., schizophrenia and autism).

\section{Sensory Contexts}

Not only can the process of recapitulating folk psychology into PP terms open up new explanatory avenues, the PP framework can be used to illuminate possible solutions to problems with current models. For example, a main issue with the aforementioned empiricist model is known as the maintenance problem (Gadsby, 2018). The starting point for this problem is the consideration that patients are exposed to significant evidence that contradicts their pathological beliefs. For example, family and carers repeatedly emphasising the patients' extreme thinness. Answering the maintenance problem involves explaining why patients maintain their false beliefs in the face of this disconfirmatory evidence.

One answer to this problem emerging from the PP literature on delusions pertains to the epistemic contexts in which such beliefs arise. Hohwy \& Rosenberg (2005) suggest that delusions arise in contexts whereby - given the kind of content of the delusional beliefreality testing via some channels of evidence is rendered exceedingly difficult. For example, delusions with a basic emotional or interoceptive content happen to be relatively private and therefore amenable only to relatively limited, private reality testing — other people's views about your own emotions and body states are mostly considered inconsequential. Similarly, theoretical background theories about the nature of emotions and body states are unlikely to dislodge the way an individual experiences them-you may know that emotions are tied to autonomous arousal but that seems inconsequential to the way you feel emotions. Perhaps then this idea can be fruitfully applied to the case of AN, helping to solve the maintenance issue in this context.

The question at hand then is whether body size is the kind of realm that is inaccessible to reality testing. First, there are a number of sources of contradictory evidence regarding body size that we believe are unlikely to sway the patients' beliefs: theoretical belief and other people's belief. Conflicting information regarding body size that arises from other people's beliefs seems unlikely to efficiently counteract patients' experience of their own body size, as the types of abnormal body size evidence posited by the basic empiricist model all plausibly include sources that are hard to address and correct on the basis of other's evidence (Gadsby, 2018). Similarly so for contradictory background beliefs. For example, patients might believe 
that someone who weighs only $35 \mathrm{~kg}$, or wears a certain size clothes should not be "overweight"-indeed clinical testimony speaks to the surprise patients experience when shopping for clothes or viewing their weight on scales (Casper et al., 1979, p. 60; Espeset et al., 2011). Yet, such forms of indirect evidence regarding body size, we posit, will be unlikely to dislodge belief which patients have more constant and direct experiential evidence for via interoceptive sensory channels.

AN beliefs about body size may thus be relatively evidentially insulated from external contradictory types of evidence, which may play some role in maintaining them. However, body size beliefs seem less obviously insulated than more typical delusional beliefs, which are often more exclusively and clearly based on relatively private emotional and interoceptive experience (e.g., made emotions, or delusions of alien control). Further, in the case of AN there does seem to be an available avenue for intermodal reality testing by the patients themselves. Intermodal reality testing occurs, for example, when one feels something that can also be seen (or vice versa). AN patients have the possibility of reality testing regarding their own body size, not through mental imagery, or self-other judgments but rather through visual perception: they may simply look at and measure their own bodies to test if indeed they are overweight or not. This form of reality testing bears on a significant issue within the AN literature: the issue of whether patients' visual experience of their bodies is veridical or not, an issue we turn to in the next section (see section 5).

The maintenance problem can also be addressed in terms of model selection, rather than direct reality testing. A belief that one is overweight may encounter plenty of contradicting evidence, but if no other equally consistent model presents itself, then the patient may be "stuck" with the false belief. This raises the question of why alternative models would have low Bayesian model evidence? One possibility has to do with complexity cost: alternative models would have to explain away the contradictory evidence and the initial evidence in support of the false belief. If these sets of data pull in different directions then the underlying model of latent causes (e.g., true weight, size, visual configuration) could be explained away only by a fairly complex model representing many interactions among causes. As such, the complexity of alternative models may partly contribute to the maintenance of false (but simple) overweight beliefs (see section 6 for further appeal to complexity).

\section{Visual Perception and Cognitive Penetrability}

... when I was younger I think I saw myself bigger in the mirror than I actually was in reality ...

With my eyes I actually saw myself as big ...

... when I look at myself in the mirror I really can't understand where I have anorexia. It's nowhere! (Espeset et al., 2011, p. 182; 184; 185)

These quotes, from three different patients, touch on a long-standing tension within the literature pertaining to the direct visual experience patients have of their own bodies. While many patients (like the above) claim to genuinely see themselves as overweight (Smeets \& Panhyusen, 1995), it has been argued that this cannot be a genuine case of misperception (Gadsby, 2017c, p. 27). Complicating matters further is the worry that positing false visual body size perception entails a kind of cognitive penetrability, whereby higher order beliefs 
influence lower level (visual) perception. There is ongoing discussion about the extent to which cognitive penetrability describes a real phenomenon or not (Firestone \& Scholl, 2013).

It seems then that the standard empiricist model is faced with a dilemma: if patients visually perceive their body size veridically, then why does this veridical perception fail to dislodge the relevant false beliefs? Alternatively, if patients see themselves as overweight, then what accounts for this distorted perception (presuming cognitive penetration is unavailable as an explanation)? Here, we would note that this dilemma is forced upon us by folk psychology's firm distinction between 'perception' and 'belief'. Such a distinction forces researchers into an explanation which refers to either perceptual or propositional belief formation processes. However, the PP framework softens the strict division between perception and belief and thereby opens the door to further explanatory resources in terms of more subtle and cyclical influences on belief formation and perceptual inference, consistent with relatively mild and uncontentious aspects of cognitive penetrability (Hohwy 2013: Ch 6).

One important insight from the PP literature pertains to the conditions under which top-down modulation of perceptual experience occurs. In the rubber hand illusion mentioned above, and many other illusions, priors modulate the incoming sensory input, giving rise to the relevant illusory percepts. What is important to note about these illusions are the conditions that facilitate top-down influence. In many illusions, perceptual conditions are relatively uncertain due to sensory noise, as when we see illusory patterns in visual noise, or due to cue ambiguity, as when congruent visuo-tactile signals conflict with proprioception in the rubber hand illusion. Uncertainty can also stem from constrained action for epistemic value, as when we cannot measure the length of lines in the Müller-Lyer illusion, or move the real hand in the rubber hand illusion. As discussed, uncertain sensory domains result in low precision estimation of the incoming prediction error and underconfident policy inference. When prediction error has low precision, priors hold more (relative) weight and are thus given greater influence in sculpting the relevant perceptual representation or shape of policies selected.

Such underlying uncertainty may also be at play in instances of self-viewing in AN. After all, as stipulated, patients are exposed to significantly noisy and ambiguous evidence regarding their own body size: some input (emanating from the experiences posited by the standard empiricist model) suggests they are overweight, while other sources (e.g. visual, testimonial) suggest they are underweight. More generally, as we speculated in Section 3, the PP framework seems to suggest that there is expected imprecision for prediction error pertaining to body size, leading to down-weighting of signals in this domain. In contexts where the expected precision of incoming prediction error is low, our perceptual representations are more highly weighted by our priors. In instances of self-viewing, then, it might be the case that for those patients far along in the disorder, the expected precision of the incoming PE will be low, causing them to form a visual representation of themselves that is more highly weighed towards their priors. If we assume that patients predict they will see an overweight body (consistent with their now well-established beliefs about being overweight), then the visual percept may be weighted towards this expectation. The PP explanation here appeals to the hierarchically bound mixture of priors and prediction errors that is fundamental to perceptual inference but in the context of the cascading dynamics of consecutive Bayesian inference.

This account coheres with anecdotal reports that, for some patients, brief perception of their own bodies as underweight often occurs in contexts where patients aren't expecting to see 
their own bodies, that is, where the context has not primed the relevant overweight priors. For example,

I remember one occasion, I was passing an open door and saw myself in the mirror, but actually, I didn't know that I saw myself. I just saw the image of a person in the mirror and thought; "Oh gosh, she is thin!" But then, when I understood that it was actually me, I didn't see me as thin anymore. But then I actually saw a glimpse of it. (Espeset et al., 2011, p.183)

Beyond the claim that, in low precision sensory contexts, perceptual content can be biased by priors, there is another explanatory avenue offered by the PP framework, which seems to cohere with evidence from the AN literature. Consider the situation of a patient with AN viewing themselves in a mirror. Such an experience will likely generate prediction error, arising from the mismatch between the incoming (thin-looking) input and the patient's expectations of their own bodies as being overweight. Agents have a number of minimization strategies available when faced with prediction error. One common way is to simply update one's priors, adjusting them such that they cohere with the incoming prediction error. If this strategy was adopted, then the patients' pathological beliefs would resolve.

However, AN patients might enact other prima facie reasonable strategies, such as action for epistemic value, in order to minimize prediction error but which transform the ordinary process of self-viewing in a mirror to one of false belief reinforcement: they might turn their visual attention away from the mirror, avoid the image altogether, or they might specifically attend to the parts of their body they judge to be unattractive (i.e. overweight). There is evidence to suggest that, when self-viewing, AN patients do visually attend toward body parts which they believe are unattractive (i.e. overweight) and that many patients avoid selfviewing altogether (Gadsby, 2018). Such strategies would enable patients to maintain their beliefs about being overweight, in the face of contradictory visual input but they would nevertheless cause the prediction error to cease. As such, the PP toolbox does offer some potential explanations for why direct visual experience of one's body size does not constitute a useful form of inter-modal reality testing: alternative ways of minimizing visually produced body size prediction error may instead come into play.

If such explanations of AN were to be on the right track, PP can begin to furnish genuine explanatory advances because the only imperative on the perceptual and cognitive system is to minimize (long-term average) prediction error, and the description of the full PP mechanism sets out several different ways this can happen. There is no independently accessible check available for the agent to the system for whether its efforts at prediction error minimization are efficient, or good for aiming at veridical beliefs. Which route to prediction error minimization is chosen is determined by the relative precisions of the different beliefs (about body size, precisions etc.) together with beliefs about what shape of model and policies may lead to the best prediction error minimization in the long-term. On an individual basis, the exact route to prediction error minimization reveals differences in priors about means and precisions including for expectations for long-term prediction error trajectories (Parr, Rees et al. 2018). Given there can be multiple different sources of evidence feeding the overweight beliefs, it is likely that there will be quite different landscapes of priors as well, associated with different trajectories for prediction error minimization and thus different presentations of AN. 
Accordingly, there is evidence of considerable individual difference both in the contents of the reported body size experiences and in the tenacity with which they are maintained. In AN, as in other mental disorders like schizophrenia, there are both between-patient differences in reported experience of body size as well as within-patient differences, as many profess to a fluctuating experience of body size.

But I really can't see any anorexia on myself. I've never had any such experiences as "maybe I'm a bit thin." Never! I always see that I'm fat. (Frida)

I've always seen that I'm thin. And that's quite unusual. I have always realized that I have a problem. (Louisa)

One day I see myself as too thin, and the next day too fat, and it can also vary from hour to hour. (Irene) (Espeset et al., 2011, p. 181)

Similarly for the characteristics of the relevant beliefs about body size. Using semi-structured interview techniques, researchers have discovered significant between-patient differences in belief characteristics such as conviction and incorrigibility (Phillipou et al., 2017).

Such diversity amongst patients may reflect diversity in the prediction error minimization strategies, including the aforementioned strategies - the sculpting of noisy prediction error by expectation (leading to visual perception of one's body as larger than reality) and the avoidance of the visual input altogether. The PP framework provides a mechanism sketch by which these different strategies can be accounted for, allowing us to account for the diversity amongst patients and understand symptom aetiology at the level of the individual. This explanation of diversity is then tied together by the hypothesis of initial problems with precision optimisation of signals normally associated with body size experience. ${ }^{1}$

\section{Model selection and simplicity}

While we have seen that the PP approach affords some genuine explanatory advances, the accounts we have considered do not quite close off the reasonable challenge that it is unclear $w h y$, at least in the longer run, patients adopt the aforementioned strategies for prediction error minimization rather than simply updating the priors pertaining to their own body size? That is, in spite of the various types of explanations PP may be able to offer, why don't patients over time construct an alternative hypothesis and slowly begin to unlearn their suboptimal inference about their body size? This question relates to the type of process that PP always returns to, which is statistical learning: the internal model is shaped through prediction error learning that should correct false inferences such that the model eventually comes to recapitulate the causal structure of the world, including the true body size of the agent themselves.

One possible answer refers to an extended history of socio-cultural influence, a theme that often recurs in AN debates. Patients are consistently exposed, through adolescence and via multiple socio-cultural channels, to unrealistic body size standards. This plausibly results in

\footnotetext{
${ }^{1}$ This furnishes a more general advantage of PP in explaining delusions (as opposed to simply the false body size beliefs of AN patients). Though often overlooked, there is significant diversity amongst patients with delusions. While some hold their beliefs with enough conviction to act on them (often with disastrous consequences), others profess to incredulity at their own belief, and within-patient vacillation (between belief and non-belief) is also evident (Coltheart, 2007).
} 
prioritising the belief that one is overweight. In the spirit of social constructivism, one might here argue that the patients are recapitulating the causal structure of the world they live in because they are picking up and inferring a powerful ideal, meshed deeply with peer behaviour, social discourse, media empires, and fashion industries. However, there is an issue with this hypothesis, in terms of the kinds of representational content socio-culturally derived priors would exhibit. It is not that patients simply believe that they are "overweight"-i.e. an evaluative claim - rather, the physical dimensions of their body are misrepresented (Gadsby, 2017a; 2018). And while socio-cultural influence may be to blame for agents believing they are overweight, it does not convey information about one's own bodily dimensions.

With PP, a relatively straightforward link can be attempted between the socio-culturally driven overweight model and the experience of body size. As the selected model, it determines the parameters for subsequent prediction error minimization. So, if the model is that one is overweight, this will generate prediction error which is sought to be minimized under that model. However, this explanation does not stick very deep, as it immediately leads to new questions: why is it that the model is not eventually de-selected once prediction error accumulates over the long-term, as body weight in fact plummets? If the explanation is on the right track, then there must be something about the selected overweight model that inoculates it against being de-selected, in spite of accumulating prediction error.

We suggest a hypothesis that is uniquely consistent with the PP account of cognition. Patients may persistently settle on the overweight model due to a bias towards simplicity. Consider for a moment the two competing models: one whereby the agent is overweight and one whereby the agent is underweight. As stated, these models will drive minimization of distinct forms of prediction error: one minimizes prediction error suggesting the agent is underweight, the other minimizes prediction error suggesting the agent is overweight. Assuming that both are more or less equal in minimizing the ongoing prediction error for body size, we must look to what other prediction error minimization advantages might be gained through adopting the (in fact false) overweight hypothesis.

First, recall that, according to the PP account, systems do not exclusively strive to minimize occurrent prediction error, but also long-term expected prediction error. This means, as mentioned earlier, that the agent considers simplicity and complexity of the competing models. This implies that there may be a preference for simpler models than more complex models even if the former are worse at relatively short-term prediction error minimization.

Following this reasoning, it may be that for patients, the overweight hypothesis is simpler than the underweight hypothesis. In assessing models' simplicity, we must consider not only the agent's model of her own body, but also her model of the causal regularities of the entire environment, including aspects of her own agency. In discussing the model selection problem - i.e. why patients choose the overweight, rather than underweight hypothesis - the AN researcher Anouk Keizer (personal communication) suggested that if patients were to accept that they had achieved their ideal size, though many of the problems in their life remain, then this would entail accepting that these perceived problems were not simply side effects of not being thin enough. In other words, patients' current model of the world exhibits significant simplicity: the achievement of ideal body size is a (single parameter) solution to a variety of psychological and social issues.

This idea - that AN patients adopt a model of the world whereby a diverse and complex set of personal problem are reduced to simply being about the patient's body size - has been 
touted regularly within the literature. For example, in reviewing a number of cognitive models of anorexia nervosa (and bulimia nervosa), Vitousek \& Hollon write:

These theories differ along a number of dimensions, but hold one important set of premises in common: that anorexics and bulimics endow weight with rich connotations, equate their personal value with the shape of their bodies, and use the regulation of weight to subserve numerous functions in their lives. (1990, p. 192). ${ }^{2}$

There are two important features of model simplicity emphasised in this quote. The first is the equation of personal value (a vastly complex multi-dimensional property) with a single feature (body shape). The second notion of simplicity highlighted pertains to action policies: the policy not to eat may be inferred at least partly because not eating is a simple policy, at least over some medium length time scale, compared to the very non-obvious and extremely complex policy one would have to single out, which could improve self-esteem and social worth. As discussed earlier, active inference implies inference of high precision policies understood as high fidelity in the mappings from actions to expected outcomes. The difference between policies can be borne out partly in terms of simplicity: the no-eat policy is much simpler than the utterly complex policies needed to changes the way the world looks upon one and the way one feels about oneself. And of course, in the initial phases of acting on the no-eat policy, there may well be positive reinforcement from the environment, which can further anchor the policy. This dual aspect of model simplicity is evident throughout Vitousek \& Holdon's discussion, as the simpler model not only "provides clear templates for evaluating daily experience," but also "prescribes a simple set of rules for seeking safety and avoiding danger [and] reduces the complexity of formulating attributions about the past and expectations for the future" (p. 193).

While there is a general propensity for simplicity for all PP processing, there will also be individual differences. Our proposal is then that it may emerge as a bias in AN. If so, we would expect such a bias to be evident in other domains too. In fact, there seems to be some evidence for this pattern. A significant body of evidence now suggests that AN patients have a higher sensitivity to the rubber hand illusion (e.g. Eshkevari et al., 2012; Keizer et al., 2014; Zopf et al., 2016). That is, when faced with the multi-modal conflict presented by the paradigm, they have a tendency for opting for the simpler model "the touch sensation and visual movement are co-located, an caused by one latent cause, and the rubber hand is mine" over the more complex "the touch sensation and visual movement are distinctly located, caused by different latent causes, and the hand is not mine". Further, susceptibility to the illusion is positively correlated with measures of eating disorder psychopathology (Eshkevari et al., 2012). This hypothesis opens the doorway to further experimentation, verifying that AN patients' model selection exhibits a greater tendency towards simplicity than neurotypical participants.

\section{Conclusion}

\footnotetext{
${ }^{2}$ Speaking to the ubiquity of this feature of the disorder amongst patients (not just models), Vitousek \& Hollon also write:
}

In the course of preparing the present paper, a number of current and former eating-disordered women were interviewed about their perceptions of the "cognitive essence" of their symptomatology; although each nominated some contributing factors not shared by the group as a whole, every one implicated the "simplification of life" as a major component of her disorder. (p. 206) 
We have discussed the PP framework and how it may explain psychopathology. Initial applications of PP appear to mainly re-describe known theories and phenomena in different terms. However, once the full and varied PP toolbox is brought to bear on psychopathological conditions, then genuine explanatory advances emerge. Here, we have focused on AN, and shown how analysis of prominent approaches (in particular, the empiricist approach) can be distinctively refined through PP, which points to the potential existence of an underlying deficit in precision optimisation, which may be implicated in unusual body size experiences. This provides a ground for a PP account addressing how the unusual beliefs about body size are maintained against counterevidence, with appeal to considerations about top-down influences across hierarchical inference, issues of model selection and model simplicity, and an attempt to understand the role of social influences on AN.

These are all explanations that become available when applying PP under the idea that agents are blind slaves to the objective function of minimizing prediction error in the long-term average by whatever tools available in the full PP toolbox. Often agents have be guided by quite abstract priors about which tools will best ensure long-term average prediction error minimization; agents might appeal to difficult-to-confirm priors about the efficiency of simplicity over complexity in a given type of context, or what signals to trust in spite of imprecision, or how to maintain the right balance between perceptual and active inference, and so on. This gives leeway for individual differences and pathological perceptual and active inference in the sense that inference becomes driven by unusual priors about, for example, the usefulness of simplicity in policy inference, the expected precision of body size experience, and the reality of ideals promulgated in social media.

Here we come up against the last charge of triviality of the PP framework, namely that it in fact does not really allow false, Bayes suboptimal inference. Rather, it allows divergent priors which explain optimal inference that appears suboptimal. This does in a sense trivialise the account of psychopathology, including AN, but can be considered a strength of PP, since it allows precise phenotyping of disorder in terms of underlying priors, making a virtue out of what is normally considered a vice of Bayesian approaches, i.e. that any behaviour can be modelled with appropriate priors.

In this perspective, the case of AN is in fact rather perplexing for the PP framework (especially as founded on the free energy principle (Friston 2010)): PP essentially says that organisms withstand destruction of their bodily boundaries and thereby death and dispersion, by minimizing their long term average of prediction error (or free energy) given a model of their expected states, which are usually conceived in terms of their homeostatic set points (heart rate, body temperature, glucose levels etc.). AN seems to rather directly lead patients to insult their homeostatic set points, in virtue of the high fidelity mapping between the action of not eating and outcomes that are in fact life-threatening - they seem to act such as to unequivocally increase probabilistic surprisal in the short and long term. The only available PP response to this is that AN patients have inferred a model of their basic homeostatic state that deviates dramatically from the expectations defining the typical human phenotype. It is however not easy to accept that our basic (homeostatic) model, against which all surprise is eventually assessed, can change as dramatically as this. This may be some kind of ultimate limitation on PP explanations. 
Aru, J., et al. (2018). "It's all in your head: Expectations create illusory perception in a dualtask setup." Consciousness and Cognition 65: 197-208.

Bogacz, R. (2017). "A tutorial on the free-energy framework for modelling perception and learning." Journal of Mathematical Psychology 76, Part B: 198-211.

Buckley, C. L., C. S. Kim, S. McGregor and A. K. Seth (2017). "The free energy principle for action and perception: A mathematical review." Journal of Mathematical Psychology 81: 55-79.

Case, L. K., Wilson, R. C., \& Ramachandran, V. S. (2012). Diminished size--weight illusion in anorexia nervosa: evidence for visuo-proprioceptive integration deficit. Experimental brain research, 217(1), 79-87.

Casper, R., Halmi, K., Goldberg, S., Eckert, E., \& Davis, J. (1979). Disturbances in body image estimation as related to other characteristics and outcome in anorexia nervosa. The British Journal of Psychiatry: The Journal of Mental Science, 134, 60-66.

Corlett, P. R., G. Horga, P. C. Fletcher, B. Alderson-Day, K. Schmack and A. R. Powers (2019). "Hallucinations and Strong Priors." Trends in Cognitive Sciences 23(2): 114-127.

Espeset, E. M., Nordbø, R. H., Gulliksen, K. S., Skårderud, F., Geller, J., \& Holte, A. (2011). The concept of body image disturbance in anorexia nervosa: an empirical inquiry utilizing patients' subjective experiences. Eating disorders, 19(2), 175-193.

Firestone, C., \& Scholl, B. (2013). 'Top-down' effects where none should be found: The El Greco fallacy in perception research. Journal of Vision, 13(9), 780.

Fletcher, P. C. and C. D. Frith (2009). "Perceiving is believing: a Bayesian approach to explaining the positive symptoms of schizophrenia." Nat Rev Neurosci 10(1): 48-58.

Friston, K. (2010). "The free-energy principle: a unified brain theory?" Nat Rev Neurosci 11: 127-138.

Friston, K. (2010). "The free-energy principle: a unified brain theory?" Nat Rev Neurosci 11(2): 127-138.

Friston, K., T. FitzGerald, F. Rigoli, P. Schwartenbeck, J. O'Doherty and G. Pezzulo (2016).

"Active inference and learning." Neurosci Biobehav Rev 68: 862-879.

Friston, K., F. Rigoli, D. Ognibene, C. Mathys, T. Fitzgerald and G. Pezzulo (2015). "Active inference and epistemic value." Cognitive Neuroscience: 1-28.

Friston, K. J., A. D. Redish and J. A. Gordon (2017). "Computational Nosology and

Precision Psychiatry." Computational Psychiatry 1: 2-23.

Gadsby, S. (2017a). Explaining body size beliefs in anorexia. Cognitive neuropsychiatry, 22(6), 495-507.

Gadsby, S. (2017b). Anorexia nervosa and oversized experiences. Philosophical

Psychology, 30(5), 594-615.

Gadsby, S. (2017c). Distorted body representations in anorexia nervosa. Consciousness and Cognition, 51, 17-33.

Gadsby, S. (2018). Self-Deception and the Second Factor: How Desire Causes Delusion in Anorexia Nervosa. Erkenntnis, 1-18.

Herbert, B.M. \& Pollatos, O. (2018). The Relevance of Interoception for Eating Behaviour and Eating Disorders, In: The Interoceptive Basis of the Mind, Eds. Manos Tsakiris \& Helena De Preester, Oxford University Press

Hohwy, J. (2013). The Predictive Mind. Oxford, Oxford University Press.

Hohwy, J. and R. Rosenberg (2005). "Unusual experiences, reality testing, and delusions of control." Mind \& Language 20(2): 141-162.

Jones, M. and B. C. Love (2011). "Bayesian fundamentalism or enlightenment? On the explanatory status and theoretical contributions of Bayesian models of cognition." Behavioral and Brain Sciences 34(4): 169-188. 
Kadriu, F., Claes, L., Witteman, C., Norré, J., Vrieze, E., \& Krans, J. (2019). Characteristics and content of intrusive images in patients with eating disorders. European Eating Disorders Review.

Keizer, A., Smeets, M. A., Postma, A., van Elburg, A., \& Dijkerman, H. C. (2014). Does the experience of ownership over a rubber hand change body size perception in anorexia nervosa patients?. Neuropsychologia, 62, 26-37.

Maher, B. A. (1974). "Delusional thinking and perceptual disorder." Journal of Individual Psychology 30: 98-113.

Marshall, L., C. Mathys, D. Ruge, A. O. de Berker, P. Dayan, K. E. Stephan and S. Bestmann (2016). "Pharmacological Fingerprints of Contextual Uncertainty." PLOS Biology 14(11): e1002575.

Montague, P. R., R. J. Dolan, K. J. Friston and P. Dayan (2012). "Computational psychiatry." Trends in Cognitive Sciences 16(1): 72-80.

Palmer, C. J., R. P. Lawson and J. Hohwy (2017). "Bayesian Approaches to Autism: Towards Volatility, Action, and Behavior." Psychological Bulletin 143(5): 521-542.

Parr, T., D. Benrimoh, P. Vincent and K. Friston (2018). "Precision and false perceptual inference." Front. Integr. Neurosci.

Parr, T. and K. J. Friston (2018). "The Anatomy of Inference: Generative Models and Brain Structure." Frontiers in Computational Neuroscience 12(90).

Parr, T., G. Rees and K. J. Friston (2018). "Computational Neuropsychology and Bayesian Inference." Frontiers in Human Neuroscience 12(61).

Phillipou, A., Mountjoy, R. L., \& Rossell, S. L. (2017). Overvalued ideas or delusions in anorexia nervosa?. Australian \& New Zealand Journal of Psychiatry, 51(6), 563-564.

Smeets, M., \& Panhuysen, G. (1995). What can be learned from body size estimation? It all depends on your theory. Eating Disorders, 3(2), 101-114.

Sterzer, P., R. A. Adams, P. Fletcher, C. Frith, S. M. Lawrie, L. Muckli, P. Petrovic, P. Uhlhaas, M. Voss and P. R. Corlett (2018). "The Predictive Coding Account of Psychosis." Biological Psychiatry 84(9): 634-643.

Van de Cruys, S., K. Evers, R. Van der Hallen, L. Van Eylen, B. Boets, L. Lee de-Wit and J. Wagemans (2014). "Precise minds in uncertain worlds: Predictive coding in autism." Psychological Review 121(4): 649-675.

Vitousek, K. B., \& Hollon, S. D. (1990). The investigation of schematic content and processing in eating disorders. Cognitive therapy and research, 14(2), 191-214.

Wald, A. (1947). "An Essentially Complete Class of Admissible Decision Functions." The Annals of Mathematical Statistics 18(4): 549-555.

Tulver, K., et al. (2019). "Individual differences in the effects of priors on perception: A multi-paradigm approach." Cognition 187: 167-177.

Zopf, R., Contini, E., Fowler, C., Mondraty, N., \& Williams, M. A. (2016). Body distortions in Anorexia Nervosa: Evidence for changed processing of multisensory bodily signals.

Psychiatry research, 245, 473-481. 